\title{
Fas-mediated apoptosis in clinical remissions of relapsing experimental autoimmune encephalomyelitis
}

\author{
Graig C. Suvannavejh, ${ }^{1}$ Mauro C. Dal Canto, ${ }^{2}$ Louis A. Matis, ${ }^{3}$ and Stephen D. Miller ${ }^{1}$ \\ ${ }^{1}$ Department of Microbiology-Immunology, and \\ ${ }^{2}$ Department of Pathology and the Interdepartmental Immunobiology Center, Northwestern University Medical School \\ and the Northwestern University Institute for Neuroscience, Chicago, Illinois 60611, USA \\ ${ }^{3}$ Alexion Pharmaceuticals, New Haven, Connecticut 06511, USA \\ Address correspondence to: Stephen D. Miller, Department of Microbiology-Immunology, Northwestern University \\ Medical School, 303 East Chicago Avenue, W213, Chicago, Illinois 60611, USA. \\ Phone: (312) 503-7674; Fax: (312) 503-1154; E-mail: s-d-miller@nwu.edu.
}

Received for publication September 29, 1999, and accepted in revised form December 8, 1999.

PLP139-51-induced experimental autoimmune encephalomyelitis (R-EAE) displays a relapsing-remitting paralytic course in female SJL mice. We investigated the role of apoptosis/activation-induced cell death (AICD) in the spontaneous recovery from acute disease. Clinical EAE was significantly enhanced in Fas (CD95/APO-1)-deficient SJL lpr/lpr mice, which displayed significantly increased mean peak clinical scores, reduced remission rates, and increased mortality when compared with their SJL $+/ l p r$ littermates. PLP139-151-specific proliferative responses were fairly equivalent in the 2 groups, but draining lymph node T cells from SJL $l p r / l p r$ mice produced dramatically increased levels of IFN- $\gamma$. Central nervous system (CNS) Fas and FasL mRNA levels in wild-type SJL $\left(H-2^{s}\right)$ mice peaked just before spontaneous disease remission and gradually declined as disease remitted. We applied the terminal deoxynucleotidyl transferase-mediated dUTP nick-end labeling (TUNEL) assay to detect apoptosis in situ in spinal cords of mice at various clinical stages of EAE. Most TUNEL ${ }^{+}$cells were found during active periods of inflammation: the acute, peak, and relapse time points. Significantly fewer apoptotic cells were observed at preclinical and remission time points. Collectively, these findings indicate that Fas-mediated apoptosis/AICD plays a major role in the spontaneous remission after the initial acute inflammatory episode and represents an important intrinsic mechanism in regulation of autoimmune responses.

J. Clin. Invest. 105:223-231 (2000).

\section{Introduction}

Experimental autoimmune encephalomyelitis (EAE) is a CD4 ${ }^{+}$Th1 cell-mediated inflammatory, demyelinating disease of the central nervous system (CNS) that can be induced by immunization of susceptible female SJL mice with PLP139-151, the immunodominant epitope on the proteolipid protein (1). This leads to a characteristic relapsing and remitting paralytic disease (2). Histological examination of the CNS reveals the presence of both a rich inflammatory mononuclear cell infiltrate and demyelination resulting from bystander activation of resident microglia and recruited, infiltrating monocytes/macrophages $(3,4)$. Disease progression is associated with the process of epitope spreading, wherein $\mathrm{T}$ cells primed to endogenous myelin epitopes are primarily responsible for mediating clinical relapses $(5,6)$. Based on the shared similarities in the disease course and histology, EAE has been used extensively as an animal model for the human disease multiple sclerosis (MS).

Although many of the salient features of the proinflammatory events of this autoimmune model have been elucidated, the exact mechanisms of disease remission remain poorly understood. A number of potential, nonmutually exclusive, mechanisms exist that may contribute to disease remission. A "cytokine switch" from a proinflammatory Th 1 to an anti-inflammatory Th 2 environment in the CNS has been postulated to immediately precede or coincide with remission from the acute clinical episode of disease. A large body of evidence from a number of laboratories (7-16), including ours (17), has shown cyclical expression of mRNA in the CNS encoding Th1 and Th2 cytokines during relapsing-remitting disease. Although it has been hypothesized that Th2 cytokines may play important roles in the downregulation of EAE, studies examining the potential regulatory role of Th2 cytokines have not always proved convincing (reviewed in ref. 18). Conflicting results on the protective roles of anti-inflammatory cytokines in EAE have been observed using mice carrying targeted deletion of various Th2 cytokines, transgenic mice overexpressing Th2 cytokines, and mice treated with recombinant Th2 cytokines (19-21). Although immune deviation may contribute to the intrinsic regulation of EAE, the process is likely to be tightly regulated and extremely complex.

Other experiments have described the existence of immunoregulatory cells that are able to directly or indirectly downregulate disease-initiating Th1 effector cells. Recovery from disease in acute, self-limiting EAE in Lewis rats and PL/J mice depends on the presence of regulatory $\mathrm{T}$ cells that are apparently able to downregulate ongoing inflammatory autoimmune response $(22,23)$. Such 
cells have been reported to be specific for either the disease-initiating antigen or for $\mathrm{T}$-cell receptor (TCR) determinants expressed on the autoreactive Th1 effector cells (24-26). These regulatory cells may simply be Th2 in nature, as it has been shown that $\mathrm{CD}^{+}$regulatory $\mathrm{T}$ cells from Lewis rats recovered from acute EAE produce TGF$\beta$ to inhibit IFN- $\gamma$ and TNF/lymphotoxin (TNF/LT) production $(27,28)$. However, Th2-like cells are not always efficient in regulating the effector activity of differentiated autoimmune Th1 effector cells and may even be capable of causing CNS pathology $(29,30)$.

An additional possibility to explain remission in R-EAE is the apoptosis/activation-induced cell death (AICD) of inflammatory CD4+ Th1 cells specific for the diseaseinducing epitope. There are multiple pathways that can lead to the activation of a death signal. Many of these pathways involve the participation and activation of evolutionarily conserved genes. One such gene, fas, encodes for the Fas receptor protein. Fas (CD95/APO-1) is a member of the TNF receptor superfamily of type 1 membrane proteins, with other family members including the lowaffinity nerve growth factor (NGF) receptor, CD27, CD30, CD40, and the p55 and p75 components of the TNF receptor (31). Fas is expressed on activated T cells, microglia, oligodendrocytes, and astrocytes (32-35). Studies using Fas-deficient $l p r$ mice have indicated a role for Fas in peripheral T-cell tolerance/deletion (36-38). Interestingly, apoptotic elimination of encephalitogenic $\mathrm{T}$ cells has also been associated with recovery from EAE in the acute Lewis rat model of disease $(39,40)$. On the basis of these observations, we sought to investigate whether Fas-mediated apoptosis played any role in disease remission in PLP139-151-induced R-EAE in the SJL mouse. Our results clearly indicate that Fas-mediated apoptosis/AICD represents an important intrinsic mechanism for regulation of ongoing autoimmune responses.

\section{Methods}

Antigens and mice. Six- to 7-week-old female SJL ( $\left.H-2^{s}\right)$ mice were purchased from Harlan Laboratories (Indianapolis, Indiana, USA) and used at 8-10 weeks of age. SJL $l p r / l p r$ mice were generated in the animal facility of Alexion Pharmaceuticals (New Haven, Connecticut, USA), with the MRL lpr/lpr mutation having been backcrossed onto the SJL background for 4 generations. These mice have been shown to develop a normal acute phase of PLP139-151-induced R-EAE (41). A breeding colony was established and maintained in the Center for Experimental Animal Research at Northwestern University (Chicago, Illinois, USA), and the mice were backcrossed 2 additional generations. Usually F6 SJL lpr/lpr males were crossed with SJL + / lpr females to yield progeny that were $50 \% \mathrm{SJL}+/ l_{\mathrm{pr}}$ and $50 \%$ SJL lpr/lpr.

Peptides. PLP139-151 (HSLGKWLGHPDKF) and PLP178-191 (NTWTTCQSIAFPSK) were synthesized using an ABI Synergy Peptide Synthesizer (Applied Biosystems, Norwalk, Conneticut, USA). Purity (> 97\%) of these peptides was confirmed by mass spectroscopy at the University of North Carolina Peptide Synthesis Core (Chapel Hill, North Carolina, USA).

Active and passive induction of EAE. For active PLP139151-induced R-EAE, each mouse received $100 \mu \mathrm{L}$ of a CFA emulsion containing $200 \mu \mathrm{g}$ of Mycobacterium tuberculosis H37Ra (Difco Laboratories, Detroit, Michigan, USA) and $50 \mu \mathrm{g}$ of PLP139-151 distributed subcutaneously over 3 spots on the dorsal flanks on day 0 . For adoptive R-EAE induction, donor SJL mice were primed in a similar fashion as for active EAE. Ten days after immunization, the inguinal, brachial, and axillary lymph nodes were removed from sensitized donors, and singlecell suspensions were prepared. The cells were adjusted to $8 \times 10^{6} / \mathrm{mL}$ in T75 flasks in complete DMEM (Sigma Chemical Co., St. Louis, Missouri, USA) supplemented with $10 \%$ FCS, $100 \mathrm{U} / \mathrm{mL}$ penicillin, $100 \mu \mathrm{g} / \mathrm{mL}$ streptomycin, $1 \mathrm{mM}$ glutamine, $1 \mathrm{mM}$ nonessential amino acids, and $5 \times 10^{-5} \mathrm{M}$ 2-mercaptoethanol (GIBCO-BRL, Grand Island, New York, USA) containing $20 \mu \mathrm{g} / \mathrm{mL}$ PLP139151 in a total volume of $30 \mathrm{~mL} /$ flask and incubated at $37^{\circ} \mathrm{C}$ in a humidified atmosphere containing $5 \% \mathrm{CO}_{2}$. Four days later, the cells were washed twice with complete media and counted, and $3 \times 10^{6}$ blast cells were transferred intraperitoneally to recipient SJL mice. Clinical scores were assessed on a $0-5$ scale as follows: 1 = limp tail or hind limb weakness (legs fall through the cage top), 2 $=$ limp tail and hind limb weakness, 3 = partial hind limb paralysis, $4=$ total hind limb paralysis, and $5=$ moribund. A relapse was defined as an increase in at least 1 clinical grade sustained for at least 2 consecutive days after the animal had previous improved at least a full clinical score and had stabilized.

T-cell proliferation and cytokine assays. Ten days after immunization, draining lymph node (LN) cells and splenocytes were isolated as already described here. For T-cell proliferation, $5 \times 10^{5}$ cells resuspended in DMEM-10 were activated with PLP139-151 and PLP178-191 in vitro in 96-well

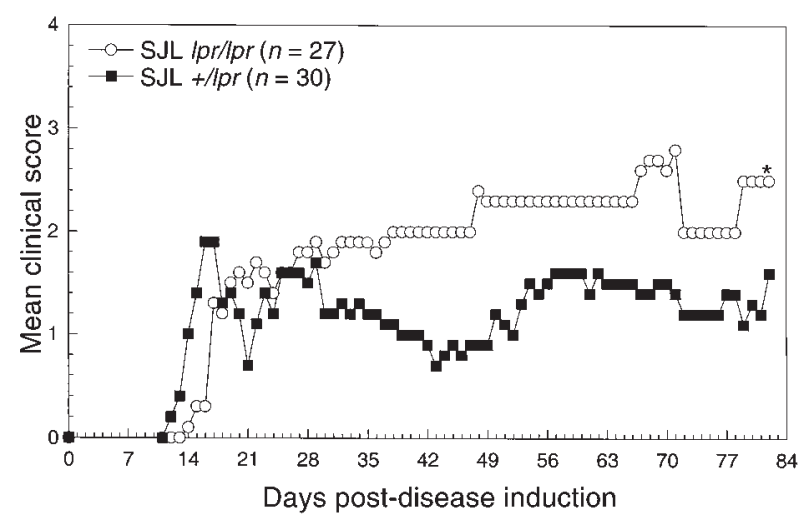

\section{Figure 1}

Clinical course of PLP139-151-induced EAE in SJL $/ p r / l p r$ and SJL $+/ / p r$ mice. EAE was induced by immunization with $50 \mu \mathrm{g}$ PLP139151 in CFA supplemented with $200 \mu \mathrm{g}$ M. tuberculosis. Mice were monitored and graded for clinical disease as described in Methods. Data represent cumulative results from separate experiments. In a few experiments $S J L+/+$ mice were also tested and showed a disease course similar to their $\mathrm{SJL}+/ /$ pr littermates. 
plates at varying concentrations. The cells were pulsed at 72 hours with $1 \mu \mathrm{Ci}\left[{ }^{3} \mathrm{H}\right]$ thymidine and harvested at 96 hours. The results are expressed as $\triangle \mathrm{CPM}=$ mean $\mathrm{CPM}$ of antigen-containing cultures - mean CPM of control cultures, and as stimulation index (SI) = mean CPM of antigen-containing cultures/mean CPM of control cultures. For cytokine assays, $5 \times 10^{6}$ cells were activated in the presence or absence of PLP139-151 in vitro in 24-well plates. Supernatants were collected after 48 hours. The concentrations of IL-2, IL-4, IL-5, and IFN- $\gamma$ present in these supernatants were quantitated with indirect ELISA assays (Endogen Inc., Woburn, Massachusetts, USA).

Histologic evaluation of demyelination. Mice were anesthetized and sacrificed by total body perfusion through the left ventricle using chilled 3\% glutaraldehyde in PBS ( $\mathrm{pH}$ 7.4). Spinal cords were dissected out and cut into 1$\mathrm{mm}$-thick segments and post-fixed in $\mathrm{OsO}_{4}$, dehydrated, and embedded in Epon (Electron Microsopy Sciences, Fort Washington, Pennsylvania, USA). Toluidine blue-stained sections from 10 segments per mouse were read and scored blinded by M. Dal Canto at Northwestern University as follows: ${ }^{+/}$, mild inflammation without demyelination; $1^{+}$, inflammation with focal demyelination; $2^{+}$, inflammation with multiple foci of demyelination; $3^{+}$, marked inflammation with bilateral converging areas of demyelination; and $4^{+}$, extensive bilateral areas of demyelination and remyelination.

Detection of Fas and Fas ligand by PCR. At various stages of clinical disease, mice were anesthetized and sacrificed by total body perfusion with PBS ( $\mathrm{pH}$ 7.4) through the left ventricle. Spinal cords were isolated by intrathecal hydrostatic pressure and homogenized by passing over a wire mesh. After washing with sterile BSS, the spinal cord homogenate was lysed with $4 \mathrm{M}$ guanidinium isothiocyanate (Life Technologies, Gaithersburg, Maryland, USA). Total RNA was isolated from the bulk spinal cords homogenate using cesium chloride extraction. After total RNA was calculated using $\mathrm{A}_{260} / \mathrm{A}_{280}$ ratios, $2 \mu \mathrm{g}$ poly $\mathrm{A}$ mRNA was reversed transcribed into cDNA using oligo (dt) primers (CLONTECH Laboratories Inc., Palo Alto, California, USA). Detection of Fas and Fas ligand signals was accomplished using the following protocol: 40 cycles of $94^{\circ} \mathrm{C}$ for 30 seconds, of $52^{\circ} \mathrm{C}$ for 30 seconds, and of $72^{\circ} \mathrm{C}$ for 30 seconds. The primers used were as follows: Fas 3', ATGATATTAGATAAAATGAT; Fas 5', ATGATGATAGATAGAT; Fas ligand 3', ATGCCATTAGCAAA; Fas ligand 5', TAGCACCTGAA. PCR products were electrophoresed on $2 \%$ agarose gels containing ethidium bromide and viewed by ultraviolet (UV) fluorescence. These signals were semiquantitatively determined after densitometric scanning. No signals were observed using control RNA. Detection of in situ apoptosis by the terminal deoxynucleotidyl transferase-mediated dUTP nick-end labeling assay. EAE mice induced by the adoptive transfer method were sacrificed by total body perfusion with PBS ( $\mathrm{pH}$ 7.4). Once spinal cords were dissected out, they were cut roughly into 8 uniform blocks about 1-mm long, embedded in OCT, and snap frozen in liquid nitrogen. Five-micron-thick frozen sections were cut on a cryostat, placed on Super-
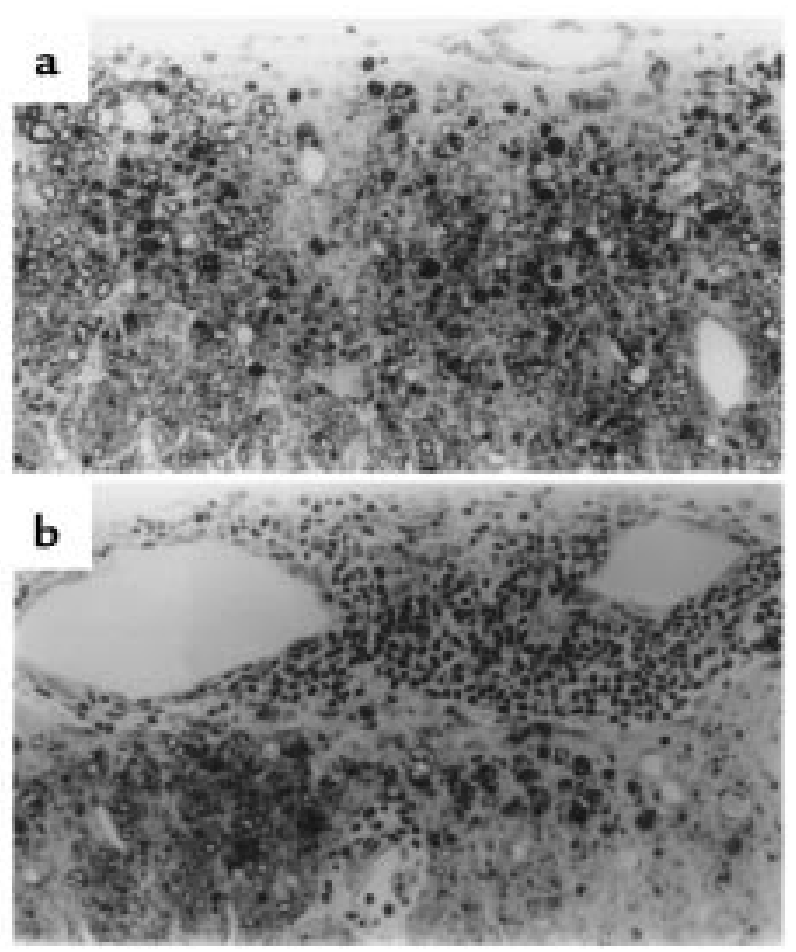

\section{Figure 2}

Histological examination of spinal cords from representative $S J L+/ l p r$ and SJL /pr/lpr mice 48 days after immunization. (a) SJL +//pr histology shows a demyelinated area with little evidence of residual inflammatory activity. Few lymphoid cells are seen in the overlying leptomeninges. (b) SJL I pr/lpr histology shows a demyelinating lesion accompanied by a striking inflammatory response. Sections are $1 \mu \mathrm{m}$ thick, Epon-embedded, and stained with toluidine blue. $\times 220$.

frost Plus slides (Fisher Scientific, Pittsburgh, Pennsylvania, USA), and stored at $-70^{\circ} \mathrm{C}$. Terminal deoxynucleotidyl transferase-mediated dUTP nick-end labeling (TUNEL) was carried out according to manufacturer's (Boehringer Mannheim GmbH, Mannheim, Germany) recommendations. Briefly, sections were fixed in $4 \%$ paraformaldehyde for 30 minutes, and permeabilized for 2 minutes in a $0.1 \%$ Triton X-100 in a $0.1 \%$ sodium citrate solution. After blocking for endogenous peroxidase activity with $0.3 \%$ hydrogen peroxide, a TUNEL reaction mixture was applied for 60 minutes at $37^{\circ} \mathrm{C}$. An anti-fluorescein peroxidase-conjugated antibody (POD) solution was used to detect the incorporation of dUTP, and slides were developed using SG (Vector Laboratories, Burlingame, California, USA) as a substrate. All washes were performed with PBS. Slides were dehydrated in isopropanol and coverslipped in Accumount. TUNEL ${ }^{+}$ immunoreactivity was detected and counted under light microscopy.

Statistical analyses. Comparison of the percentage of animals showing clinical disease or relapses between any 2 groups of mice was done by $\chi^{2}$ using Fisher's exact probability. Comparisons of the mean day of onset of relapse and mean peak disease severity between any 2 groups of mice were analyzed by the Student's $t$ test. $P$ values less than 0.05 were considered significant. 


\section{Results}

EAE is enhanced in Fas-deficient SJL mice. We first compared the pattern of PLP139-151-induced clinical disease in SJL $l p r / l p r$ mice and control SJL + / lpr littermates. SJL +/lpr mice are highly susceptible to PLP139-151-induced REAE, exhibiting a characteristic relapsing-remitting disease course that is very reproducible (Figure 1). However, SJL $l p r / l p r$ displayed a chronic progressive clinical disease course that was significantly more severe than the control SJL +/lpr littermates (Figure 1). Both groups underwent a similar acute phase of disease; however, unlike the SJL +/lpr controls, Fas-deficient SJL mice generally failed to remit from the acute clinical disease episode. This separation in the 2 disease courses remained evident for as long as 80 days after priming and was observed in 3 separate experiments.

The enhanced postacute clinical disease in Fas-deficient SJL mice became more evident when additional disease parameters were examined (Table 1). Although the disease incidence was slightly reduced in SJL $l p r / l p r$ mice compared with heterozygous littermates $(21 / 27$ [78\%] versus 29/30 [97\%]; $P=0.047$ ), of those mice that displayed clinical disease, the mean peak clinical score was significantly worse in $l p r / l p r$ mice (4.05 vs. 3.03; $P<0.001$ ), and this difference was sustained throughout the 82-day observation period. More striking was the fact that the number of mice remitting from acute disease was dramatically decreased in Fas-deficient animals compared with +/- littermates (7/21 [33\%] vs. 29/29 [100\%]; $P<$ $0.001)$, and the mortality rate was significantly increased $(12 / 21$ [57\%] vs. $1 / 29$ [3\%]; $P<0.001)$.

Histological analysis performed 7 weeks after immunization reveals an interesting difference in CNS histology between SJL Fas-deficient mice and their Fas heterozygote littermates of chronically affected mice.

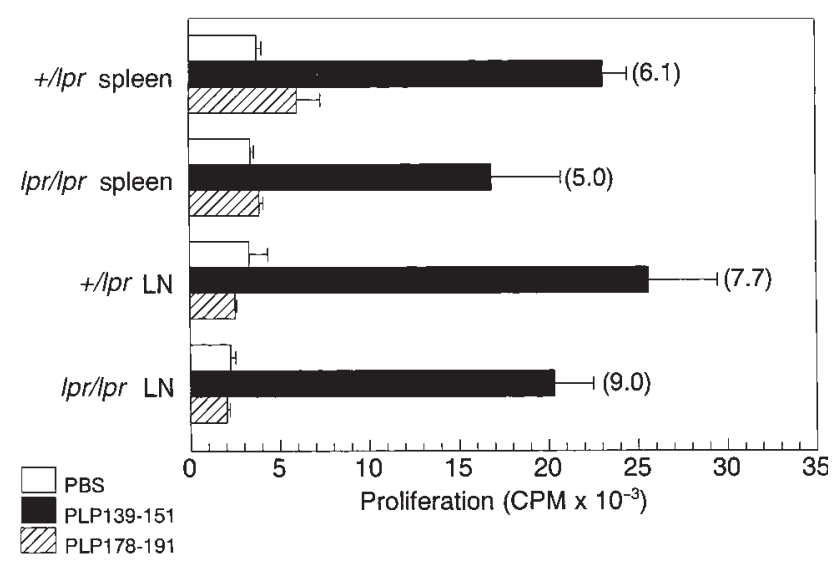

\section{Figure 3}

Myelin peptide-specific proliferative responses of PLP139151-primed SJL +/lpr and SJL Ipr/lpr mice. A total of $5 \times 10^{5}$ splenic and draining $L N$ cells harvested 15 days after immunization from the indicated groups were stimulated in vitro with $50 \mu \mathrm{M}$ PLP139-151, PLP178-191, or PBS. Cultures were pulsed with $\left[{ }^{3} \mathrm{H}\right] \mathrm{TdR}$ at 72 hours and harvested 24 hours thereafter. Numbers in parentheses represent PLP139-151-specific stimulation indices (SI). SI values greater than 3.0 are considered significant.

\section{Table 1}

Comparison of clinical scores between SJL $/ p r / p r$ and SJL +/lpr mice primed with PLP139-151/CFA

\begin{tabular}{|c|c|c|}
\hline \multirow[b]{2}{*}{ Disease parameter ${ }^{A}$} & \multicolumn{2}{|c|}{ Genotype } \\
\hline & $\mathrm{SJL}+/ / p r$ & SJL Ipr/lpr \\
\hline Disease incidence & $29 / 30$ & $21 / 27^{B}$ \\
\hline Mean peak clinical score & $3.03 \pm 0.14$ & $4.05 \pm 0.28$ \\
\hline $\begin{array}{l}\text { Mean clinical score } \\
\text { at end of the experiment }\end{array}$ & $1.37 \pm 0.21$ & $2.67 \pm 0.45^{C}$ \\
\hline Remission incidence & $29 / 29$ & $7 / 21^{c}$ \\
\hline Mortality incidence & $1 / 29$ & $12 / 21^{\mathrm{C}}$ \\
\hline
\end{tabular}

ADisease parameters are defined as follows: disease incidence $=$ number of clinically affected animals/total number of immunized mice; mean peak clinical score $=$ mean clinical score for affected animals at the peak of disease; remission incidence $=$ number of mice exhibiting a clinical remission $/$ total number of affected mice; and mortality incidence $=$ number of mice dying/total number of affected mice. ${ }^{\mathrm{B}} P<0.05,{ }^{C} P<0.001$.

Although representative spinal cord sections from both SJL + / lpr and $l p r / p r$ mice demonstrated equal demyelination, there was a tremendous increase in the number of inflammatory mononuclear cells in the spinal cords of Fas lpr/lpr mice (Figure 2, a and b).

Collectively, these results indicate that Fas-mediated mechanisms play a relatively minor contributory role in mediating acute clinical disease symptoms in the SJL mouse. However, the results clearly indicate that Fasmediated apoptosis plays a major role in interactions leading to the intrinsic regulation of ongoing inflammatory disease in relapsing-remitting EAE.

Comparison of PLP139-151-specific immune responses in SJL $+/ l p r$ and SJL $l p r / p r$ mice. To address whether the exacerbated chronic disease course in Fas-deficient mice might be due to a qualitative or quantitative difference in myelin peptide-specific cellular responses, in vitro proliferative assays and cytokine ELISA assays were performed. Draining LN cell and splenocyte populations isolated from PLP139-151-primed SJL $l p r / l p r$ mice and their $+/ l p r$ littermates showed equivalent proliferative responses 15 days after sensitization (Figure 3). Responses directed toward a secondary but non-cross-reactive PLP178-191 epitope involved in epitope spreading in this model (5) were not significantly above background values at this early point in disease. Supernatants from these PLP139151-stimulated cultures were also analyzed for the presence of Th1 and Th2 cytokines. With the exception of slightly enhanced production by splenocytes isolated from $+/ l p r$ mice, IL-2 levels as detected by ELISA were found to be comparable (Figure 4a). Of particular interest, IFN- $\gamma$ production from draining LN cells of $l p r / l p r$ mice was significantly higher than those levels found in the other groups (Figure 4b). Excessive production of IFN- $\gamma$ may contribute to the increased severity of chronic EAE observed in SJL lpr/lpr mice. IL-4 and IL-5 were not detected from any of the cultures (data not shown).

CNS Fas and FasL mRNA expression and TUNEL $L^{+}$ immunoreactivity correlate with relapsing-remitting disease progression. To attempt to assess what role, if any, apoptosis might play in remission in R-EAE in wild-type SJL mice, we examined CNS levels of Fas and FasL mRNA expres- 
sion at various stages of clinical disease. Semiquantitative RT-PCR revealed that Fas and FasL mRNA expression reached a maximal level in mice undergoing acute clinical disease, and then gradually declined to low levels as the disease progressed through to remission (Figure 5). Fas L mRNA expression declined more quickly than did the expression of Fas message. Nevertheless, mRNA levels of both proapoptotic molecules always remained detectable. To evaluate the degree of apoptosis occurring in situ in the CNS, TUNEL assays were performed on spinal cord samples taken during various clinical stages of disease (Table 2, Figure 6). Preclinically, little apoptosis was detected (Figure 6a); yet, as animals began to show symptoms of clinical disease, $\mathrm{TUNEL}^{+}$cells became readily apparent, remaining high at the peak of acute clinical disease (Figure 6b). The numbers of apoptotic cells were quite substantially decreased as animals underwent disease remission, but then increased to high levels at the peak of the primary relapse, just before entering the second remission (Table 2). These results suggest that peak apoptotic activity precedes remission from both the acute disease and the primarily relapse by 3-4 days and is consistent with a role for AICD of effector Th1 cells in mediating intrinsic regulation of the progressive autoimmune disease process. As expected, very few TUNEL ${ }^{+}$cells were evident in the spinal cord of SJL $l p r / l p r$ mice with chronic EAE (Table 2, Figure 6c).

\section{Discussion}

We investigated the potential role of apoptosis/AICD of myelin epitope-specific T cells as a mechanism for disease remission in relapsing-remitting EAE in the SJL mouse. The data clearly indicate that the disease course of EAE in clinically affected Fas-deficient SJL mice was dramatically enhanced when compared with Fas $+/ / p r$ littermate controls. The incidence of disease remission in Fas-deficient mice was significantly decreased (33\% vs. $100 \%$; $P<0.001)$ compared with controls, and, at the same time, the incidence of mortality from progressive disease was significantly greater $(57 \%$ vs. $3 \%$; $P<0.001)$ than the control mice (Table 1). Collectively, these findings suggest that the absence of Fas allows activated myelin-specific, disease-initiating cells to escape peripheral and/or CNS deletion, to remain in the CNS target organ, and to cause prolonged and enhanced inflammation. This was evident by histological analysis of spinal cords from SJL $l p r / l p r$ mice, which revealed persistent inflammation that is distinctively unusual for such a late time point (Figure 2) and by the finding of few apoptotic cells in the CNS of these mice (Figure 6, Table 2). This possibility is also supported by the enhanced levels of IFN- $\gamma$ produced by PLP139-151-specific draining LN T cells of Fas-deficient mice (Figure 4).

To determine the potential role of Fas-mediated apoptosis in the intrinsic regulation of R-EAE in wild-type SJL mice, we examined the pattern of Fas and FasL mRNA expression in the CNS of mice at varying stages of the relapsing-remitting disease process. Neither message was detected in spinal cord samples before disease onset. However, both messages could be detected during disease a

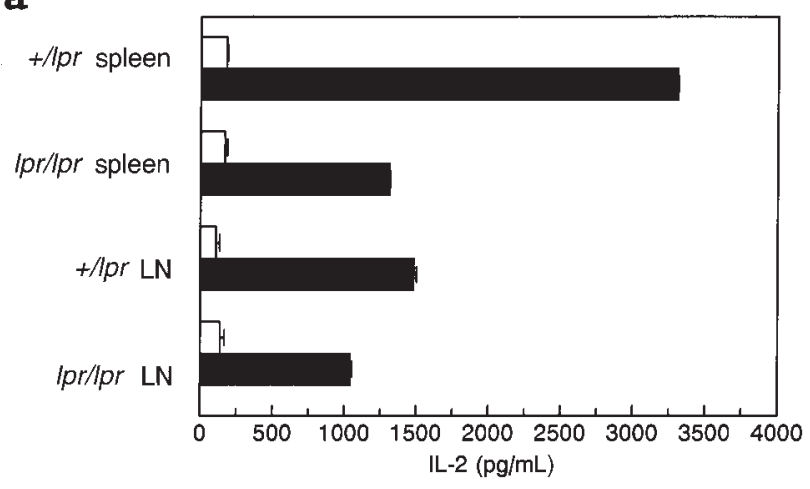

b

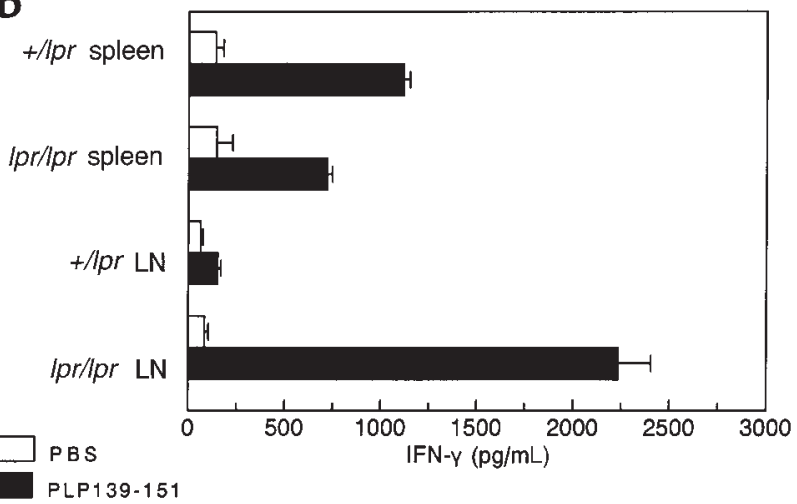

Figure 4

PLP139-151-specific proinflammatory cytokine secretion from SJL $+/ / p r$ and SJL Ipr/lpr mice at day 15 after immunization. Culture supernates of PLP139-151 stimulated splenic and draining LN cells were collected at 48 hours after culture initiation, and the levels of IL-2 (a) and IFN- $\boldsymbol{\gamma}(\mathbf{b})$ were determined by ELISA. IL-4 and IL-5 were not detected from any of the cultures (data not shown).

onset and remained detectable as the disease progressed through the peak of acute clinical phase and then fell dramatically as mice entered full remission from acute disease. TUNEL immunohistochemical analysis was used to determine further the relationship between the level of apoptosis occurring in situ in the CNS target tissue and remission. High numbers of apoptotic foci were detected during the inflammatory period surrounding the peak of acute disease, then dropped off dramatically as the mice entered remission, and increased again as the animals

\section{Table 2}

TUNEL quantitation of apoptotic foci in T4 and L1 spinal cord sections during the progression of EAE in SJL mice

\begin{tabular}{lc}
\hline Clinical stage $^{\mathrm{A}}$ & No. of apoptotic foci \\
Preclinical & $1.2 \pm 0.5$ \\
Onset/acute & $74.2 \pm 11.9$ \\
Peak of acute & $62.9 \pm 6.2$ \\
Early remission & $36.8 \pm 12.5$ \\
Late remission & $36.5 \pm 15.2$ \\
Relapse & $73.3 \pm 19.7$ \\
lpr/lpr chronic & $6.17 \pm 3.1$
\end{tabular}

ADisease stages are defined as indicated in the legend to Table 1. ${ }^{\mathrm{B} M e a n}$ number of TUNEL ${ }^{+}$cells was determined for 4-6 sections of the T4 and L1 spinal cord regions. 


\section{Figure 5}

Expression of Fas and FasL mRNA in the spinal cord of wild-type SJL mice at various stages of clinical course of R-EAE by RT-PCR. R-EAE was induced by the intraperitoneal adoptive transfer of $3 \times 10^{6}$ PLP139-151-specific SJL T-cell blasts into naive wild-type SJL mice. Spinal cords from at least 2 animals were harvested at the indicated disease stages, and $2 \mu \mathrm{g}$ total mRNA was reverse transcribed into cDNA. A total of $5 \mu \mathrm{L}$ cDNA was loaded for detection of HPRT, a housekeeping gene, and Fas; $10 \mu \mathrm{L}$ cDNA was used for FasL. The 2 early remission time points shown correspond to 1 and 2 days after the peak of acute disease.

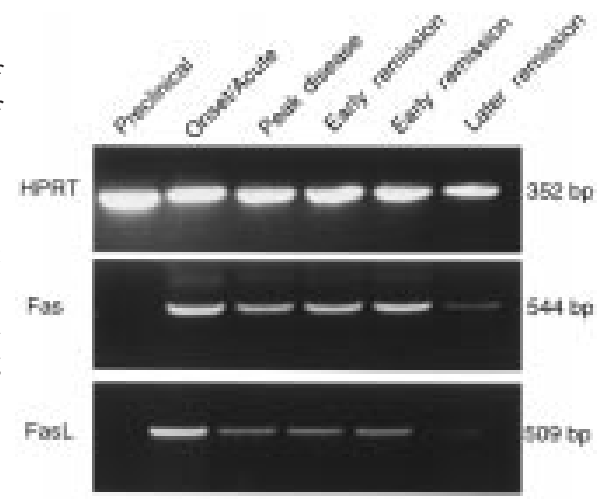

There have been conflicting observations relevant to the potential role of Fas in autoimmune disease. Mice carrying the $l p r$ mutation, including the SJL strain used in these studies, develop obvious signs of spontaneous autoimmunity as evidenced by enlarged lymph nodes, splenomegaly, the presence of circulating

underwent the initial clinical relapse. These data also indicate a correlation between apoptosis and disease remission. It could be argued that if Fas-mediated apoptosis is a major intrinsic mechanism regulating EAE, AICD should be most evident during remission. However, viewing AICD in light of its role in maintaining immune homeostasis, it is more logical for apoptosis to occur during acute inflammatory periods when there are many activated $\mathrm{T}$ cells expressing Fas in the CNS parenchyma (33). Clinical remission, the manifestation of any peripheral and/or CNS inflammatory cell deletion that may take place would not become clinically evident until several days later. Interestingly the greatest number of apoptotic cells in myelin basic protein-induced (MBPinduced) EAE in the Lewis rat is also found at the peak of acute disease (39). Collectively, these findings suggest, in the SJL mouse, that Fas-mediated apoptotic events are most prevalent during periods of CNS inflammatory damage and primarily serve to limit the degree of inflammation via AICD of autoreactive T cells. It is relevant that we have reported a dramatic downregulation of mRNA for the Th1-derived proinflammatory cytokines IFN- $\gamma$, TNF- $\alpha$, and LT in the CNS that ensues shortly after the peak of apoptosis (17).

Though our data indicate that the numbers of apoptotic foci correlate positively with acute inflammatory stages of disease just before clinical remission, we have yet to determine, quantitatively or qualitatively, the exact nature of the cells undergoing apoptosis. It has been reported that effector $\mathrm{CD}^{+} \mathrm{T}$ cells but not target cells, e.g., oligodendrocytes, bear markers of apoptosis in the CNS of mice with adoptive EAE (33). However, it has been reported that oligodendrocytes in active MS lesions express Fas (42). and microglia have been reported to be particularly susceptible to apoptosis (43). Our preliminary experiments using double-label immunofluorescence indicate that $\mathrm{CD}^{+} \mathrm{T}$ cells and $\mathrm{F} 4 / 80^{+}$ macrophages/microglia constitute the majority of apoptotic cells in the CNS parenchyma of SJL mice. Nevertheless, it will be important to identify accurately and to quantitate all cell populations undergoing apoptosis in the CNS, i.e., resident microglia, infiltrating macrophages, locally activated or recently recruited $\mathrm{T}$ cells, oligodendrocytes, and astrocytes, which are able to process and present neuroantigens to $\mathrm{T}$ cell lines and hybridomas in vitro (44). autoantibodies, and an abnormal accumulation of $\mathrm{CD}^{+}{ }^{+} \mathrm{CD} 4^{-} \mathrm{CD} 8^{-}$double negative (DN) T cells (45). This accumulation of DN T cells appears to arise from a defect in peripheral deletion that is Fas mediated $(37,38,46,47)$. Conceivably, Fas-mediated apoptosis may play contradictory roles in inflammatory autoimmune diseases. On one hand, as suggested by its critical role in peripheral T-cell deletion and in apoptotic death of activated T cells (48), Fas may play a downregulatory role in clinical remission of autoimmune disease. This view is supported by the current results that indicate that Fas-mediated AICD of activated autoreactive myelin-specific $\mathrm{T}$ cells and/or macrophages/microglia that have infiltrated the CNS plays a major role in intrinsic regulatory mechanisms used by the SJL host to limit autoimmune CNS destruction. A growing body of evidence points to a potential downregulatory role for apoptosis. In MBP-induced EAE in the Lewis rat, which undergoes an acute, self-limiting clinical paralytic disease, apoptotic elimination of CNS-infiltrating encephalitogenic V $\beta 8.2^{+} \mathrm{MBP}$-specific T cells has been shown to correlate with the resolution of inflammation and onset of clinical recovery $(39,49)$. Also, Chen et al. (50) have also reported that that deletion of MBP-specific encephalitogenic $T$ cells in the brain, using a transgenic model of acute EAE, is associated with recovery. And, finally, it has recently been shown that infiltrating $\mathrm{CD}^{+}$ T cells in adoptive EAE exhibit a significant increase in Fas and FasL expression (33) (also our unpublished results) making them especially prone to apoptosis.

Alternatively, Fas may play an important role in mediating autoimmune tissue destruction. Upregulation of FasL on activated autoreactive $\mathrm{T}$ cells, together with upregulation of Fas on resident cells in the target organ derived proinflammatory cytokines, may lead to direct target destruction. Thus, Fas may play a crucial role in mediating the destructive aspects of $\mathrm{T}$ cell-mediated autoimmunity, and one would predict that Fas-deficient mice may show reduced autoimmune tissue destruction. A potential role for Fas-mediated tissue destruction in autoimmune diabetes and MS/EAE is supported by several recent studies. Two separate reports have established that Fas-deficient NOD $l p r / l p r$ mice are resistant to both spontaneous and adoptively transferred diabetes $(51,52)$. In addition, a positive correlation between pancreatic $\beta$ cell destruction and Fas expression has been reported of an autoimmune disease induced by exposure to Th1- 
using a syngeneic islet transplant model (53). More relevant to the current data, recent reports have also shown that clinical symptoms of EAE were significantly reduced in both Fas- and FasL-deficient B10.PL $\left(H-2^{u}\right)$ and C57BL/6 $\left(H-2^{b}\right)$ when compared with wild-type controls $(54,55)$. Both disease incidence and severity, as well as mean peak clinical disease scores, were significantly diminished in these animals. Although the present results and an earlier study (41) observed a slight, but significant, decrease in disease incidence in Fas-deficient SJL mice ( $77 \%$ vs. $100 \%$ in $+/$ - littermates), our results do not support a major role for Fas-mediated effector events in mediating clinical disease in the SJL mouse. Preliminary studies examining the pathogenesis of Theiler's murine encephalomyelitis virus-induced demyelinating disease (TMEV-IDD), a CD4 ${ }^{+} \mathrm{T}$ cell-dependent virus model of MS, in Fas-deficient SJL mice also show enhanced clinical disease (unpublished observations). Other reports also support our hypothesis. Fas-deficient C57BL/6 $l \mathrm{pr} / \mathrm{lpr}$ mice are fully susceptible to adoptive EAE induced by the transfer of wild-type encephalitogenic T-cell clones (56). Collectively, these findings suggest that Fas/FasLmediated cell death in the CNS does not play an integral role in the effector phase of acute EAE.

To determine further the exact role for Fas in regulating the relapsing-remitting disease in SJL mice, we are currently performing adoptive transfer experiments involving reciprocal transfers of PLP139-151-specific T cells from wild-type and SJL $l p r / p r$ donors into wild-type and SJL $l p r / l p r$ recipients. On the basis of the current findings in active R-EAE, we might expect that EAE in SJL mice to be exacerbated in disease initiated by $l p r / l p r T$ cells. The recent literature again reveals some uncertainly regarding the contribution of Fas in EAE. SabelkoDownes et al. have recently reported that although EAE could be induced by both the transfer of wild-type B10.PL MBP-specific T cells into B10.PL $/ p r / l p r$ recipients and by the transfer of MBP-specific lpr/lpr cells into wild-type recipients, the diseases in both cases were significantly reduced in incidence and severity (57). These results suggest that Fas may be critical in the induction and progression of EAE. Interestingly, these authors also found that the transfer of MBP-specific FasL-deficient B10.PL gld cells into gld recipients resulted in a dramatic exacerbation of disease. Similar results have also been reported by Dittel et al., whereby MBP-specific wild-type TCR transgenic cells mediated an exacerbated disease when transferred into gld recipients (58). These 2 findings illustrate a potential role for FasL in both disease pathogenesis and disease recovery. These apparently contradictory results indicate that more work remains to be done to elucidate the exact role of Fas/FasL interactions in EAE. Although the disparate effects of the lpr mutation in EAE in the different mouse strains may be due to the particular immunizing neuroantigens that were used (MOG35-55 and guinea pig MBP versus PLP139-151 in our studies), as well as the use of pertussis toxin (not used in our experiments), it is probable that the main reason for the apparent disparate outcomes between our findings and the results reported by others is the genetic differences in the strains of mice used, i.e., B10.PL $\left(H-2^{u}\right)$ and B6 $\left(H-2^{b}\right)$ versus SJL $\left(H-2^{s}\right)$. Indeed, it has been reported that multiple background genes influence the incidence and severity of lymphoaccumulation and histopathologic manifestations of systemic autoimmunity in mice homozygous for the $l p r$ mutation (59). SJL mice are highly autoimmune-prone mice and redundant apoptotic-initiating stimuli, i.e., TNF/TNF-receptor-mediated apoptosis and/or nonapoptotic mechanisms of tissue destruction, may play
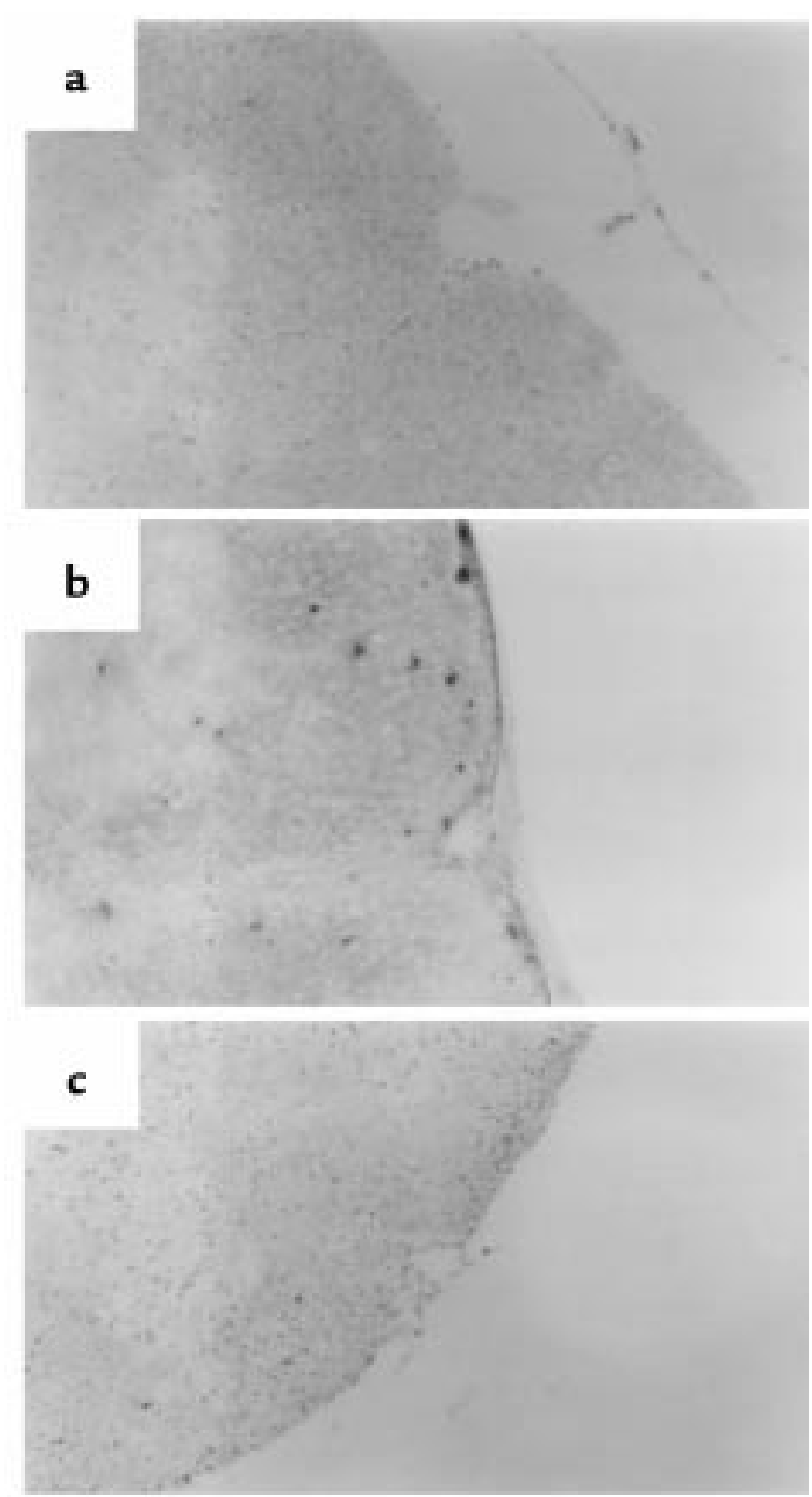

\section{Figure 6}

TUNEL analysis of in situ apoptosis in spinal cords of wild-type and SJL / pr/lpr mice. Sections show representative sections of the upper lumbar region and were viewed at $\times 10$ by light microscopy: (a) section from a wild-type SJL mouse during the preclinical stage of disease showing few TUNEL ${ }^{+}$cells; (b) section from a wild-type SJL mouse at the peak of acute disease showing considerable numbers of TUNEL ${ }^{+}$cells; and (c) section from an SJL $/ p r / l p r$ mouse during chronic-progressive EAE showing few TUNEL+ cells. 
the major effector role in CNS demyelination in this mouse strain.

In summary, our results support the hypothesis that apoptosis/AICD, involving the Fas-FasL system, is critically involved in mediating clinical remission in relapsing-remitting EAE. It is likely that Fas-FasL interactions lead to the deletion of disease-initiating, autoreactive $\mathrm{T}$ cells in the CNS and/or the peripheral immune system. Thus, these findings may be important in gaining a fuller understanding of some of the intrinsic immunoregulatory mechanisms involved in MS and may help in the design of therapeutic agents or protocols for treatment of this disease.

\section{Acknowledgments}

This work was supported by US Public Health Service/National Institutes of Health grants NS26543 (to S.D. Miller), NS30871 (to S.D. Miller), and NS13011 (to M.C. Dal Canto) and by National Multiple Sclerosis Society grant RG2893 (to M.C. Dal Canto).

1. Kennedy, M.K., et al. 1990. Inhibition of murine relapsing experimental autoimmune encephalomyelitis by immune tolerance to proteolipid protein and its encephalitogenic peptides. J. Immunol. 144:909-915.

2. McRae, B.L., Kennedy, M.K., Tan, L.J., Dal Canto, M.C., and Miller, S.D. 1992. Induction of active and adoptive chronic-relapsing experimental autoimmune encephalomyelitis (EAE) using an encephalitogenic epitope of proteolipid protein. J. Neuroimmunol. 38:229-240.

3. Brown, A., McFarlin, D.E., and Raine, C.S. 1982. Chronologic neuropathology of relapsing experimental allergic encephalomyelitis in the mouse. Lab. Invest. 46:171-185.

4. Raine, C.S., Mokhtarian, F., and McFarlin, D.E. 1984. Adoptively transferred chronic relapsing experimental autoimmune encephalomyelitis in the mouse. Neuropathologic analysis. Lab. Invest. 51:534-546.

5. McRae, B.L., Vanderlugt, C.L., Dal Canto, M.C., and Miller, S.D. 1995. Functional evidence for epitope spreading in the relapsing pathology of experimental autoimmune encephalomyelitis. J. Exp. Med. 182:75-85.

6. Yu, M., Johnson, J.M., and Tuohy, V.K. 1996. A predictable sequential determinant spreading cascade invariably accompanies progression of experimental autoimmune encephalomyelitis: a basis for peptide-specific therapy after onset of clinical disease. J. Exp. Med. 183:1777-1788.

7. Kennedy, M.K., Torrance, D.S., Picha, K.S., Mohler, K.M. 1992. Analysis of cytokine mRNA expression in the central nervous system of mice with experimental autoimmune encephalomyelitis reveals that IL-10 mRNA expression correlates with recovery. J. Immunol. 149:2496-2505.

8. Issazadeh, S., Ljungdahl, A., Hojeberg, B., Mustafa, M., and Olsson, T. 1995. Cytokine production in the central nervous system of Lewis rats with experimental autoimmune encephalomyelitis: dynamics of mRNA expression for IL-10, interleukin-12, cytolysin, tumor necrosis factor alpha, and tumor necrosis beta. J. Neuroimmunol. 61:205-212.

9. Tanuma, N., et al. 1997. Competitive PCR quantification of pro- and anti-inflammatory cytokine mRNA in the central nervous system during autoimmune encephalomyelitis. J. Neuroimmunol. 73:197-206.

10. Racke, M.K., et al. 1991. Prevention and treatment of chronic relapsing experimental allergic encephalomyelitis by transforming growth factorbeta 1. J. Immunol. 146:3012-3017.

11. Rott, O., Fleischer, B., and Cash, E. 1994. Interleukin-10 prevents experimental allergic encephalomyelitis in rats. Eur. J. Immunol. 24:1434-1440.

12. Racke, M.K., et al. 1994. Cytokine-induced immune deviation as a therapy for inflammatory autoimmune disease. J. Exp. Med. 180:1961-1966.

13. Johns, L.D., Flanders, K.C., Ranges, G.E., and Sriram, S. 1991. Successful treatment of experimental allergic encephalomyelitis with transforming growth factor-beta 1. J. Immunol. 147:1792-1796.

14. Racke, M.K., et al. 1992. Evidence of endogenous regulatory function of transforming growth factor-beta 1 in experimental allergic encephalomyelitis. Int. Immunol. 4:615-620.

15. Inobe, J.I., Chen, Y., and Weiner, H.L. 1996. In vivo administration of IL4 induces TGF-beta-producing cells and protects animals from experimental autoimmune encephalomyelitis. Ann. NY Acad. Sci. 778:390-392.

16. Shaw, M.K., et al. 1997. Local delivery of interleukin 4 by retrovirus-transduced $\mathrm{T}$ lymphocytes ameliorates experimental autoimmune encephalomyelitis. J. Exp. Med. 185:1711-1714.

17. Begolka, W.S., Vanderlugt, C.L., Rahbe, S.M., and Miller, S.D. 1998. Differential expression of inflammatory cytokines parallels progression of central nervous system pathology in two clinically distinct models of multiple sclerosis. J. Immunol. 161:4437-4446.

18. Begolka, W.S., and Miller, S.D. 1998. Cytokines as intrinsic and exogenous regulators of pathogenesis in experimental autoimmune encephalomyelitis. Res. Immunol. 149:771-781.

19. Bettelli, E., et al. 1998. IL-10 is critical in the regulation of autoimmune encephalomyelitis as demonstrated by studies of IL-10- and IL-4-deficient and transgenic mice. J. Immunol. 161:3299-3306.

20. Wyss-Coray, T., Borrow, P., Brooker, M.J., and Mucke, L. 1997. Astroglial overproduction of TGF-beta 1 enhances inflammatory central nervous system disease in transgenic mice. J. Neuroimmunol. 77:45-50.

21. Cannella, B., Gao, Y.L., Brosnan, C., and Raine, C.S. 1996. IL-10 fails to abrogate experimental autoimmune encephalomyelitis. J. Neurosci. Res. 45:735-746.

22. Sun, D., Qin, Y., Chluba, J., Epplen, J.T., and Wekerle, H. 1988. Suppression of experimentally induced autoimmune encephalomyelitis by cytolytic T-T cell interactions. Nature. 332:843-845.

23. Lider, O., Beraud, E., Reshef, T., Friedman, A., and Cohen, I.R. 1989. Vaccination against experimental autoimmune encephalomyelitis using a subencephalitogenic dose of autoimmune effector T cells. II. Induction of a protective anti-idiotypic response. J. Autoimmun. 2:87-99.

24. Vandenbark, A.A., Hashim, G., and Offner, H. 1989. Immunization with a synthetic T-cell receptor V-region peptide protects against experimental autoimmune encephalomyelitis. Nature. 341:541-544.

25. Kumar, V., and Sercarz, E.E. 1993. The involvement of T cell receptor peptide-specific regulatory CD4+ $\mathrm{T}$ cells in recovery from antigeninduced autoimmune disease. J. Exp. Med. 178:909-916.

26. Kumar, V., Stellrecht, K., and Sercarz, E. 1996. Inactivation of T cell receptor peptide-specific $\mathrm{CD} 4$ regulatory $\mathrm{T}$ cells induces chronic experimental autoimmune encephalomyelitis (EAE). J. Exp. Med. 184:1609-1618.

27. Karpus, W.J., and Swanborg, R.H. 1991. CD4+ suppressor cells inhibit the function of effector cells of experimental autoimmune encephalomyelitis through a mechanism involving transforming growth factor-beta. J. Immunol. 146:1163-1168.

28. Stevens, D.B., Gould, K.E., and Swanborg, R.H. 1994. Transforming growth factor-beta 1 inhibits tumor necrosis factor- alpha/lymphotoxin production and adoptive transfer of disease by effector cells of autoimmune encephalomyelitis. J. Neuroimmunol. 51:77-83.

29. Khoruts, A., Miller, S.D., and Jenkins, M.K. 1995. Neuroantigen-specific Th2 cells are inefficient suppressors of experimental autoimmune encephalomyelitis induced by effector Th1 cells. J. Immunol. 155:5011-5017.

30. Lafaille, J.J., et al. 1997. Myelin basic protein-specific T helper 2 (Th2) cells cause experimental autoimmune encephalomyelitis in immunodeficient hosts rather than protect them from the disease. J. Exp. Med. 186: 307-312.

31. Ware, C.F., VanArsdale, S., and VanArsdale, T.L. 1996. Apoptosis mediated by the TNF-related cytokine and receptor families. J. Cell. Biochem. 60:47-55

32. Brunner, T., et al. 1995. Cell-autonomous Fas (CD95)/Fas-ligand interaction mediates activation-induced apoptosis in T-cell hybridomas. Nature. 373:441-444.

33. Bonetti, B., Pohl, J., Gao, Y.L., and Raine, C.S. 1997. Cell death during autoimmune demyelination: effector but not target cells are eliminated by apoptosis. J. Immunol. 159:5733-5741.

34. Malipiero, U., et al. 1997. Myelin oligodendrocyte glycoprotein-induced autoimmune encephalomyelitis is chronic/relapsing in perforin knockout mice, but monophasic in Fas- and Fas ligand-deficient lpr and gld mice. Eur. J. Immunol. 27:3151-3160.

35. Becher, B., D’Souza, S.D., Troutt, A.B., and Antel, J.P. 1998. Fas expression on human fetal astrocytes without susceptibility to fas-mediated cytotoxicity. Neuroscience. 84:627-634.

36. Watanabe-Fukunaga, R., Brannan, C.I., Copeland, N.G., Jenkins, N.A., and Nagata, S. 1992. Lymphoproliferation disorder in mice explained by defects in Fas antigen that mediates apoptosis. Nature. 356:314-317.

37. Russell, J.H., Rush, B., Weaver, C., and Wang, R. 1993. Mature T cells of autoimmune lpr/lpr mice have a defect in antigen-stimulated suicide. Proc. Natl. Acad. Sci. USA. 90:4409-4413.

38. Singer, G.G., and Abbas, A.K. 1994. The fas antigen is involved in peripheral but not thymic deletion of $\mathrm{T}$ lymphocytes in $\mathrm{T}$ cell receptor transgenic mice. Immunity. 1:365-371.

39. Tabi, Z., McCombe, P.A., and Pender, M.P. 1994. Apoptotic elimination of $V$ beta $8.2+$ cells from the central nervous system during recovery from experimental autoimmune encephalomyelitis induced by the passive transfer of $\mathrm{V}$ beta $8.2+$ encephalitogenic $\mathrm{T}$ cells. Eur. J. Immunol. 24:2609-2617.

40. White, C.A., McCombe, P.A., and Pender, M.P. 1998. The roles of Fas, Fas Ligand and bcl-2 in T cell apoptosis in the central nervous system in experimental autoimmune encephalomyelitis. J. Neuroimmunol. 82:47-55.

41. Elliott, E.A., et al. 1996. Treatment of experimental encephalomyelitis 
with a novel chimeric fusion protein of myelin basic protein and proteolipid protein. J. Clin. Invest. 98:1602-1612.

42. D’Souza, S.D., et al. 1996. Multiple sclerosis: Fas signaling in oligodendrocyte cell death. J. Exp. Med. 184:2361-2370.

43. White, C.A., McCombe, P.A., and Pender, M.P. 1998. Microglia are more susceptible than macrophages to apoptosis in the central nervous system in experimental autoimmune encephalomyelitis through a mechanism not involving Fas (CD95). Int. Immunol. 10:935-941.

44. Tan, L.J., Gordon, K.B., Mueller, J.P., Matis, L.A., and Miller, S.D. 1998. Presentation of proteolipid protein epitopes and B7-1-dependent activation of encephalitogenic T cells by IFN-gamma-activated SJL/J astrocytes. J. Immunol. 160:4271-4279.

45. Cohen, P.L., and Eisenberg, R.A. 1991. Lpr and gld: single gene models of systemic autoimmunity and lymphoproliferative disease. Annu. Rev. Immunol. 9:243-269.

46. Van Parijs, L., Ibraghimov, A., and Abbas, A.K. 1996. The roles of costimulation and Fas in $\mathrm{T}$ cell apoptosis and peripheral tolerance. Immunity. 4:321-328.

47. Fuss, I.J., et al. 1997. Characteristic T helper $2 \mathrm{~T}$ cell cytokine abnormalities in autoimmune lymphoproliferative syndrome, a syndrome marked by defective apoptosis and humoral autoimmunity. J. Immunol. 158:1912-1918.

48. Lenardo, M.J. 1991. Interleukin-2 programs mouse $\alpha \beta$ T lymphocytes for apoptosis. Nature. 353:858-861.

49. Pender, M.P., McCombe, P.A., Yoong, G., and Nguyen, K.B. 1992. Apoptosis of alpha beta $\mathrm{T}$ lymphocytes in the nervous system in experimental autoimmune encephalomyelitis: its possible implications for recovery and acquired tolerance. J. Autoimmun. 5:401-410.

50. Chen, Y., Hancock, W.W., Marks, R., Gonnella, P., and Weiner, H.L. 1999. Mechanisms of recovery from experimental autoimmune encephalomyelitis: $\mathrm{T}$ cell deletion and immune deviation in myelin basic protein $\mathrm{T}$ cell receptor transgenic mice. J. Neuroimmunol. 82:149-159.

51. Itoh, N., et al. 1997. Requirement of Fas for the development of autoimmune diabetes in nonobese diabetic mice. J. Exp. Med. 186:613-618.

52. Chervonsky, A.V., et al. 1997. The role of Fas in autoimmune diabetes. Cell. 89:17-24.

53. Suarez-Pinzon, W., et al. 1999. Beta-cell destruction in NOD mice correlates with Fas (CD95) expression on beta-cells and proinflammatory cytokine expression in islets. Diabetes. 48:21-28.

54. Sabelko, K.A., Kelly, K.A., Nahm, M.H., Cross, A.H., and Russell, J.H. 1997. Fas and Fas ligand enhance the pathogenesis of experimental allergic encephalomyelitis, but are not essential for immune privilege in the central nervous system. J. Immunol. 159:3096-3099.

55. Waldner, H., Sobel, R.A., Howard, E., and Kuchroo, V.K. 1997. Fas- and FasL-deficient mice are resistant to induction of autoimmune encephalomyelitis. J. Immunol. 159:3100-3103.

56. Clark, R.B., Grunnet, M., and Lingenheld, E.G. 1997. Adoptively transferred EAE in mice bearing the lpr mutation. Clin. Immunol. Immunopathol. 85:315-319.

57. Sabelko-Downes, K.A., Cross, A.H., and Russell, J.H. 1999. Dual role for Fas ligand in the initiation of and recovery from experimental allergic encephalomyelitis. J. Exp. Med. 189:1195-1205.

58. Dittel, B.N., Merchant, R.M., and Janeway, C.A., Jr. 1999. Evidence for Fas-dependent and Fas-independent mechanisms in the pathogenesis of experimental autoimmune encephalomyelitis. J. Immunol. 162:6392-6400.

59. Vidal, S., Kono, D.H., and Theofilopoulos, A.N. 1998. Loci predisposing to autoimmunity in MRL-Fas lpr and C57B/6-Faslpr mice. J. Clin. Invest. 101:696-702. 